\title{
Tailoring Intermolecular Interactions for Efficient Room-Temperature Phosphorescence from Purely Organic Materials in Amorphous Polymer Matrices**
}

\author{
Min Sang Kwon, Dongwook Lee, Sungbaek Seo, Jaehun Jung, and Jinsang Kim*
}

\begin{abstract}
Herein we report a rational design strategy for tailoring intermolecular interactions to enhance room-temperature phosphorescence from purely organic materials in amorphous matrices at ambient conditions. The built-in strong halogen and hydrogen bonding between the newly developed phosphor G1 and the poly(vinyl alcohol) (PVA) matrix efficiently suppresses vibrational dissipation and thus enables bright room-temperature phosphorescence (RTP) with quantum yields reaching $24 \%$. Furthermore, we found that modulation of the strength of halogen and hydrogen bonding in the G1-PVA system by water molecules produced unique reversible phosphorescence-to-fluorescence switching behavior. This unique system can be utilized as a ratiometric water sensor.
\end{abstract}

Purely organic materials that show room-temperature phosphorescence (RTP) are attractive alternatives to organometallic phosphors. They have a few potential advantages over their organometallic counterparts: Organometallic compounds are expensive and conceivably toxic owing to the presence of metals, and are also intrinsically unstable in the case of high-energy blue emitters. ${ }^{[1]}$ However, unfortunately, purely organic RTP has been only observed from particular organic molecules ${ }^{[2-4]}$ and under stringent conditions ${ }^{[5-8]}$ because triplet excitons are not commonly generated in light-element organic materials, and those few are mostly consumed through radiationless processes, such as vibrational dissipation and oxygen-mediated quenching, under ambient conditions. ${ }^{[9,10]}$

The key factors for the construction of efficient purely organic RTP materials are 1) the promotion of both singletto-triplet and triplet-to-singlet intersystem crossing, and

[*] Dr. M. S. Kwon, ${ }^{[+]}$Prof. Dr. J. Kim

Department of Materials Science and Engineering

University of Michigan (USA)

E-mail: Jinsang@umich.edu

D. Lee, ${ }^{[+]}$S. Seo, J. Jung, Prof. Dr. J. Kim

Macromolecular Science and Engineering

University of Michigan (USA)

Prof. Dr. J. Kim

Department of Chemical Engineering

University of Michigan (USA)

Prof. Dr. J. Kim

Department of Chemistry, University of Michigan (USA)

$\left.{ }^{+}\right]$These authors contributed equally.

$[* *$ This research was partly supported by a Samsung GRO grant. D.L. was partly supported by a fellowship from LG Chemicals.

porting information for this article is available on the WWWW under http://dx.doi.org/10.1002/anie.201404490.
2) suppression of the nonradiative quenching processes from the triplet to the ground state. Within this context, several research groups have shown that "crystal" ${ }^{[11-14]}$ and/or "engineered crystal" ${ }^{[15]}$ can be used to induce efficient purely organic RTP. The rigidity and oxygen-barrier properties of crystals suppress the nonradiative deactivation pathways of triplet excitons, and, as in our previous study, the directed intermolecular halogen bonding promotes the intersystemcrossing processes by enhancing spin-orbit coupling. ${ }^{[12,19]}$

Amorphous purely organic RTP systems, for example, phosphors embedded in polymers and/or amorphous solids, would be more promising for practical applications, such as organic light-emitting diodes (OLEDs), solid-state lightning, and sensors. However, bright organic RTP is a challenging goal in the amorphous phase because active vibration/ diffusion motions, for example, $\beta$ and $\alpha$ transitions, are generally inevitable in an amorphous matrix under ambient conditions. This unfavorable amorphous environment promotes the vibrational dissipation of long-lived triplets of embedded phosphors, thus resulting in the complete quenching of phosphorescence. ${ }^{[16,17]}$ Therefore, it remains a demanding and challenging task to devise a way of minimizing diffusional motion in an amorphous matrix to obtain highly emissive organic RTP. Recently, Adachi and co-workers developed a unique amorphous purely organic RTP system by adopting a highly deuterated fluorene derivative as a phosphorescent guest and its steroid analogue as a host matrix. ${ }^{[18]}$ The considerable rigidity of the steroidal matrix combined with the heavy deuteration of phosphors synergistically suppressed the vibrational dissipation of long-lived triplets. Our research group recently demonstrated RTP in an amorphous organic material by embedding 2,5-dihexyloxy-4bromobenzaldehyde (Br6A), an organic phosphor, in isotactic poly(methyl methacrylate) (iPMMA), a rigid polymer matrix, without $\beta$ relaxation. ${ }^{[19]}$ Although these strategies demonstrated that the restriction of vibration of both the matrix and phosphors is critically important for amorphous purely organic RTP, a more generally applicable and practically useful strategy to enable efficient suppression of vibration is highly desired.

Herein, we describe our rational and systematic approach to the development of an efficient amorphous purely organic RTP system. To minimize both the diffusion motion of the matrix and the vibration of phosphors, we intentionally included two types of strong intermolecular noncovalent interactions in the material (Scheme 1). First, intermolecular halogen bonds between phosphors were introduced to facilitate intersystem-crossing processes as well as to suppress the vibration of phosphors. Second, strong intermolecular 


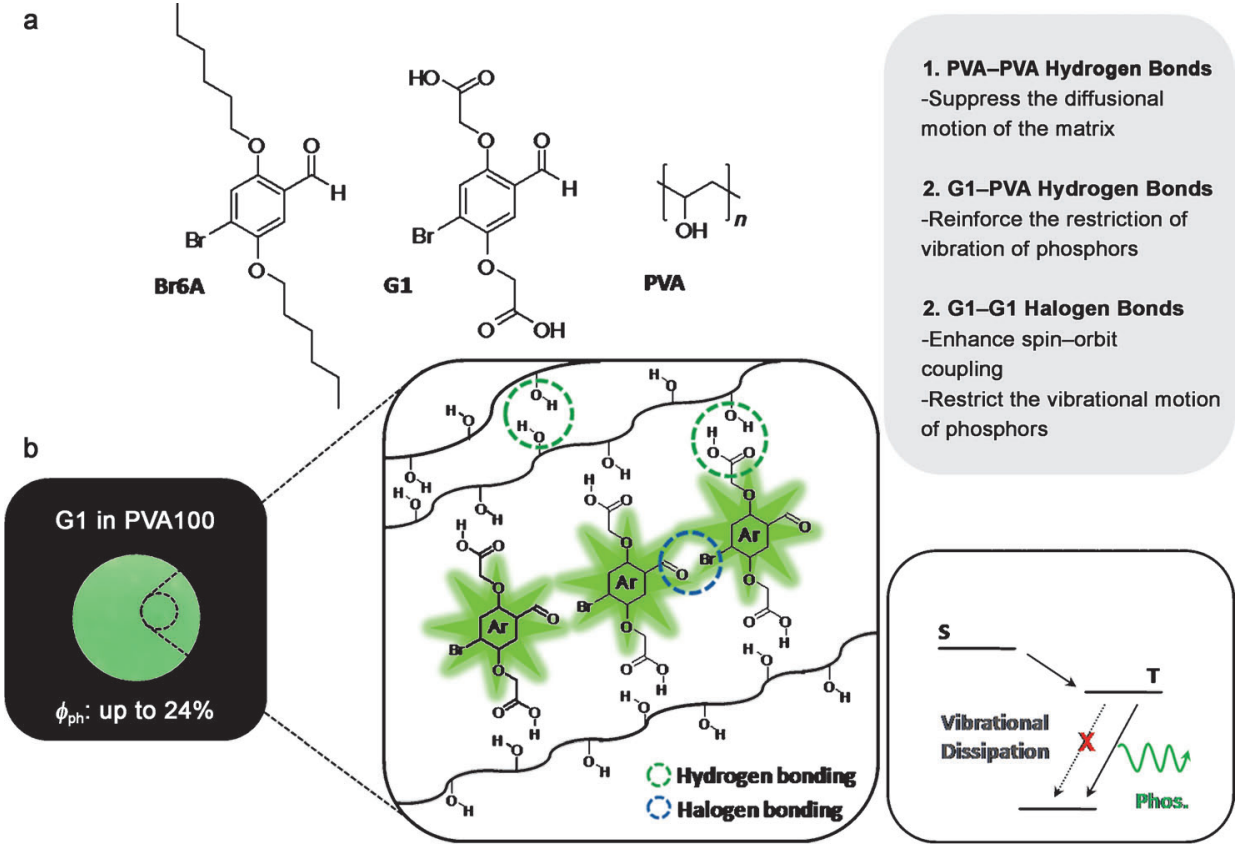

Scheme 1. a) Chemical structures of Br6A, G1, and PVA. b) Phosphorescence image of $G 1$ embedded in PVA100 under UV light ( $\lambda=365 \mathrm{~nm}$; left) and schematic illustration of phosphorescence processes in the G1-PVA composite (right).

hydrogen bonds (H-bonds) between matrix polymers as well as between matrix polymers and phosphors were incorporated to restrict the vibration/diffusion motion of the matrix and phosphors more efficiently. Thus, we designed a new organic phosphor (G1; Scheme 1a) with a bromoaldehyde core and carboxylic acid side chains as a dopant. The bromoaldehyde core was expected to provide the structural basis for strong halogen bonding in the assembly as well as for triplet-exciton generation. The carboxylic acid periphery was expected to enable the formation of strong $\mathrm{H}$-bonds between the phosphor and the selected amorphous matrix, that is, poly(vinyl alcohol) (PVA), which has H-bonding capability.

As anticipated, we observed strong RTP from G1-PVA blend films with $\Phi_{\mathrm{ph}}$ values of up to $24 \%$ at ambient conditions, which is three times as high as that of previously reported Br6A-iPMMA blend amorphous films ( $\Phi_{\mathrm{ph}}=$ $7.5 \%)$. We also found that modulation of the H-bonding strength in the G1-PVA system by water molecules produced unique reversible "phosphorescence-to-fluorescence" switching behavior. Unlike traditional organometallic phosphors, our purely organic phosphors emit RTP not from an isolated species but from molecular assemblies because of the necessary intermolecular halogen bonding to enhance spinorbit coupling. Thus, if external stimuli can break up the molecular assembly, the efficiency of spin-orbit coupling drops, thus resulting in fluorescence rather than phosphorescence emission. On the basis of this interesting stimuliresponsive change in optical properties, we could successfully demonstrate reversible direct writing of a fluorescent watermark (Figure 6a) and moisture sensing (see Figures S4 and S5 in the Supporting Information).

We first examined the solution-state luminescence properties of G1. An aqueous solution of G1 under ambient conditions showed blue fluorescence emission at $460 \mathrm{~nm}$ (Figure 1) with a quantum yield of $11.5 \%$ and a lifetime of $2.18 \mathrm{~ns}$ (see Figure S1). We did not observe any phosphorescence emission from an aqueous solution of G1 even under oxygen-free conditions. However, when the solution was cooled to $77 \mathrm{~K}$, strong green phosphorescence emission at $510 \mathrm{~nm}$ was detected, with a long lifetime of $5.9 \mathrm{~ms}$ (see Figure S2). The solution was diluted to $10^{-5} \mathrm{M}$ to rule out intermolecular interactions between molecules. At this cryogenic temperature, solvents are frozen, which greatly suppresses the vibrational dissipation of triplets and thus allows phosphorescence emission.

A G1-doped PVA film was prepared by simple drop casting from an aqueous solution of $\mathrm{G} 1$ with $80 \%$ hydrolyzed PVA (denoted PVA $80 ; 1 \mathrm{wt} \%$ of $\mathrm{G} 1$ in PVA80). PVA is a polyhydroxy polymer and can efficiently form intermolecular $\mathrm{H}$-bonds with various polymers ${ }^{[20]}$ and functionalized small-molecular dopants ${ }^{[21,22]}$ as well as interand intramolecular H-bonds with PVA itself at room temperature. ${ }^{[23]}$ These unique characteristics of PVA enabled the use of PVA as a host material for the newly synthesized phosphor, G1. The resulting thin film showed strong phosphorescence with $\lambda_{\max }=530 \mathrm{~nm}$ and only a soft shoulder in the fluorescence region at around $430 \mathrm{~nm}$ (Figure 2a). The gated photoluminescence (PL) spectrum (Figure 2a) and long lifetime of $4.7 \mathrm{~ms}$ (see Figure S3) clearly confirmed that the emission at $530 \mathrm{~nm}$ was phosphorescence. Notably, the phosphorescence quantum efficiency reached $13 \%$, which is

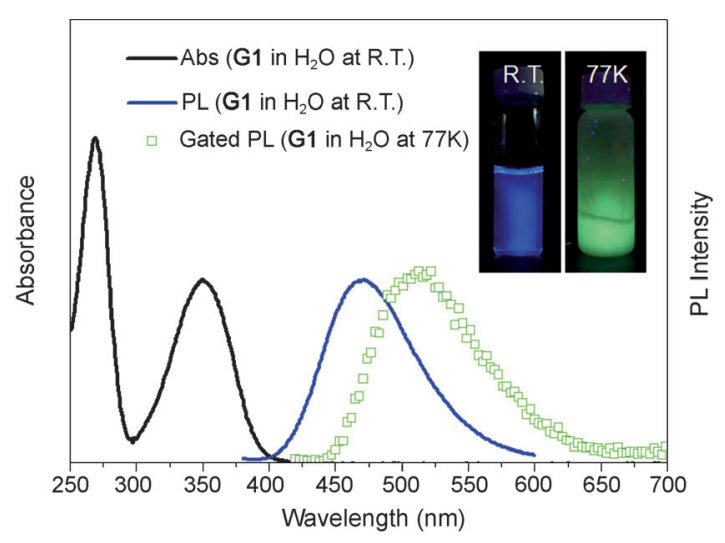

Figure 1. UV/Vis absorption (black line), PL (blue line), and gated PL spectra (green squares) of an aqueous solution of $\mathrm{G} 1$. The inset shows photoluminescence images of an aqueous solution of $\mathrm{G} 1$ at room temperature and at $77 \mathrm{~K}$. 

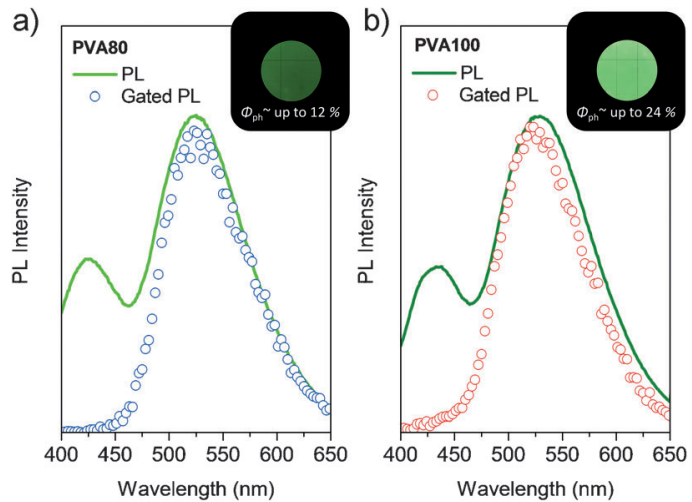

Figure 2. PL and gated PL spectra of a) a PVA80 thin film doped with $1 \mathrm{wt} \% \mathrm{Gl}$ and b) a PVA100 thin film doped with $1 \mathrm{wt} \% \mathrm{Gl}$. Insets show phosphorescence images of the corresponding G1-doped PVA80 and PVA100 thin films.

approximately 1.5 times that of the Br6A-iPMMA thin film. We believe that this large enhancement in phosphorescence quantum efficiency can be attributed to the strong self-Hbonding of the rigid polymer matrix in combination with the restriction of vibrational motion by the intermolecular $\mathrm{H}$ bonds between PVA80 and G1.

We further improved the intermolecular H-bonding efficiency by using $100 \%$ hydrolyzed PVA (denoted PVA100). PVA100 has $25 \%$ more H-bonding sites than PVA80, which lead to more efficient interpolymer H-bonding and consequentially enhanced rigidity of the PVA matrix. Furthermore, the increased number of $\mathrm{H}$-bonding sites also provide more opportunities for the formation of $\mathrm{H}$-bonds between PVA100 and G1. Thus, PVA100 was expected to suppress rotational and vibrational motion more efficiently than PVA80. Figure $2 \mathrm{~b}$ displays PL and gated PL spectra as well as a photograph of the PVA100 thin film with embedded G1. As expected, the film produced significantly enhanced phosphorescence emission with a quantum yield of up to $24 \%$. This result clearly demonstrates that our intermolecular H-bonding strategy to suppress vibration dissipation is a very effective method to achieve bright organic RTP in the amorphous state.

We tested the Br6A-PVA80 system to deconvolute the role of the H-bonding interactions of G1-PVA and PVAPVA on phosphorescence. Br6A molecules cannot form $\mathrm{H}$ bonds with the PVA matrix because of the absence of a carboxylic acid periphery. In contrast, PVA-PVA H-bonds are retained in the Br6A-PVA system. Br6A-PVA80 thin films (1 wt \% Br6A) were fabricated from a solution of PVA 80 and Br6A in $N, N$-dimethylformamide (DMF) by means of solution drop casting. The Br6A-doped PVA80 thin films produced green phosphorescence at room temperature. However, the phosphorescence quantum efficiency was only $2.2 \%$, thus indicating that the $\mathrm{H}$-bonds between $\mathrm{G} 1$ and PVA play a critical role in restricting the vibration and movement of G1 in the PVA matrix.

We investigated the effect of the polymer matrix to further prove our hypothesis. We prepared poly(vinyl acetate) (PVAc)/PVA80 blend films with various blend ratios. (Since PVA100 is soluble only in water, but PVAc is not soluble in

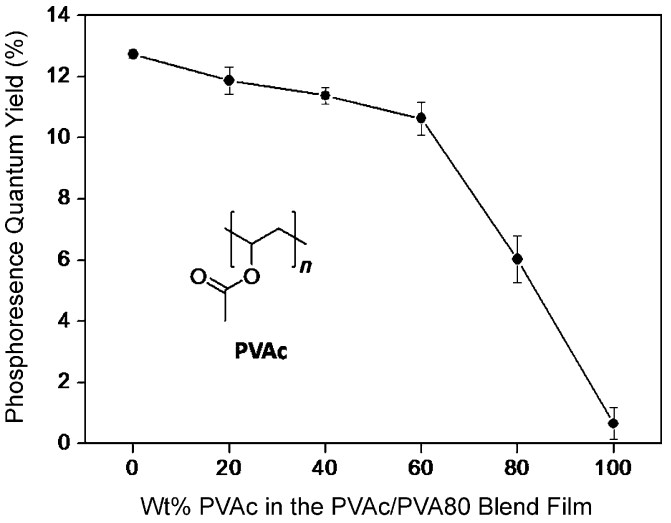

Figure 3. Phosphorescence quantum yield of $G 1$ in PVAc/PVA 80 blend films with various blend ratios. The chemical structure of PVAc is also shown.

water, we used PVA80 instead, and dissolved PVA80 with PVAc in DMF.) Each polymer blend was dissolved with G1 and used to make thin-layer PVAc/PVA80 films with embedded G1. The phosphorescence quantum yield of G1 deceased from 13 to $0.5 \%$ as the weight percent of PVAc increased from 0 to $100 \%$ (Figure 3). Unlike the hydroxy functional group of PVA, the acetyl moiety of PVAc is a weak H-bonding acceptor. Although PVAc can form H-bonds with PVA and G1, the H-bonding strength is weaker than that possible with PVA. Moreover, PVAc cannot form H-bonds with itself because it does not have a H-bond-donor group. Thus, an increase in PVAc content leads to a drop in both the strength and number of $\mathrm{H}$-bonds between matrix polymers as well as between polymers and G1, which results in significant activation of vibrational dissipation and consequent phosphorescence quenching.

Figure 4 a shows the effect of the doping concentration of G1. An optimum G1 concentration was identified to be $1 \mathrm{wt} \%$ relative to the polymer mass. At a higher concen- a)

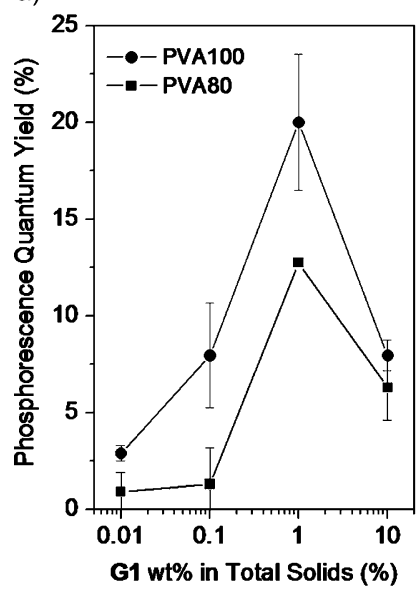

b)

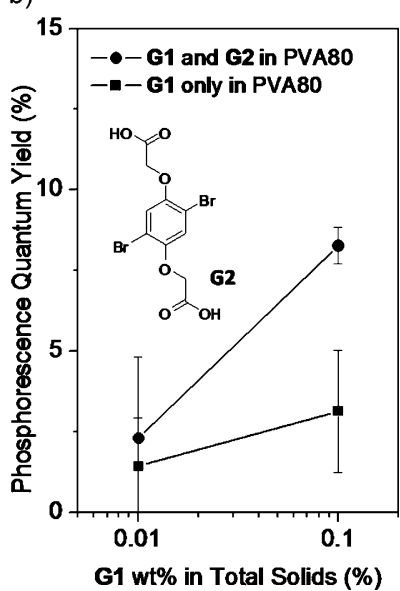

Figure 4. a) Phosphorescence quantum yield at different phosphor concentrations for G1-doped PVA80 and PVA100. b) Phosphorescence quantum yield of G1-doped PVA80 and G1/G2-doped (1:10,w/w) PVA80. The chemical structure of $G 2$ is also shown. 
tration than $1 \mathrm{wt} \%$, self-quenching caused the observed low emission yield. ${ }^{[12,19]}$ At a very low concentration, the number of G1 molecules is too small to enable the necessary halogen bonding between G1 molecules for efficient spin-orbit coupling, thus resulting in the observed minimal phosphorescence quantum efficiency. This hypothesis could be proved by additionally doping the G1-PVA80 system with G2, a dibromo analogue of G1. (Unlike G1, G2 is not soluble in water. Thus, we used PVA 80 as the polymer matrix and DMF as the solvent). G2 does not produce RTP even in the PVA matrix by itself, because G2 lacks the triplet-generating benzaldehyde structure. However, it can readily form strong halogen bonds with G1, which significantly enhances spinorbit coupling and suppresses the vibration of G1. Figure 4b clearly shows the effect of the codopant on the phosphorescence quantum yield. When the 0.01 and $0.1 \mathrm{wt} \%$ G1PVA80 system was codoped with an additional 0.1 and $1 \mathrm{wt} \%$ of $\mathrm{G} 2$, the phosphorescence quantum yield increased from 0.5 and $2 \%$ to 2 and $9 \%$, respectively.

The intensity of phosphorescence emission of the G1PVA system was strongly dependent on the air humidity (Figure 5a). As the humidity increased, the phosphorescence intensity decreased linearly (Figure $5 \mathrm{~b}$ ), whereas the fluorescence emission peak at $437 \mathrm{~nm}$ remained unchanged. We can deduce that at higher humidity, more water is absorbed by the G1-PVA system, and that the absorbed water can efficiently break the H-bonds between PVA molecules and between G1 and PVA. Breaking of the H-bonds consequently enhances the motion of PVA and vibrational motion of G1, thus resulting in the significant decrease observed in the phosphorescence quantum efficiency. In contrast, the fluorescence intensity is not dependent on humidity because the radiative decay rate of singlet excitons is much faster than that of triplet excitons.

Interestingly, the phosphorescence of G1-PVA was readily converted into blue fluorescence when water was dropped onto the G1-PVA film. On the basis of this behavior, we successfully demonstrated the use of our material as an "optical recording medium": The characters "UM" were written on the surface of the G1-PVA film (10 wt \% G1/100\% hydrolyzed PVA) with a water-soaked pen (Figure 6a; see also Movie 1 in the Supporting Information). Unlike the response to humidity, water not only breaks the PVA-PVA and PVA-G1 Hbonds, but also breaks the halogen bonds between phosphors and thus finally dissolves the matrix and phosphors. The dissolved phosphors now emit strong fluorescence, not phosphorescence (Figure 6b). This unique system can be utilized as a ratiometric water sensor. We demonstrated several
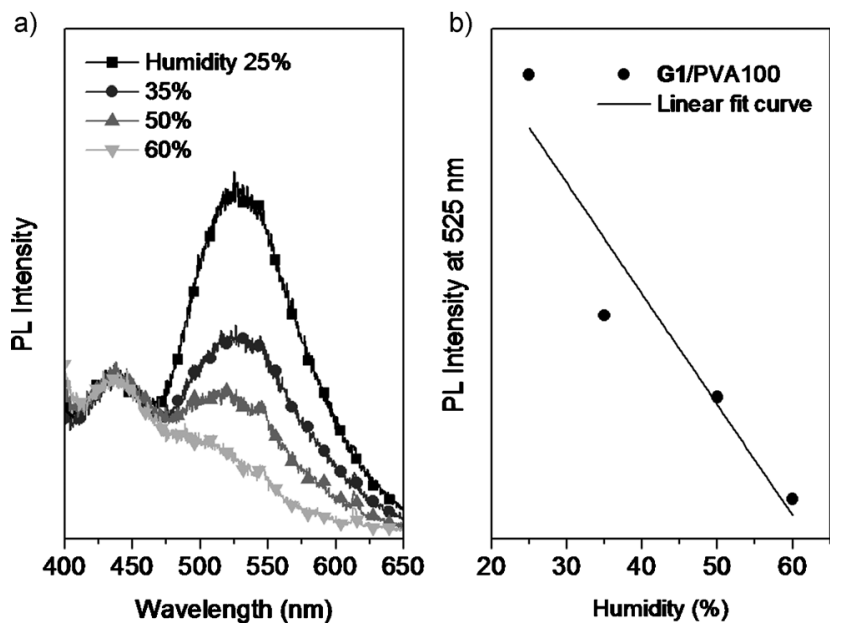

Figure 5. a) PL spectra of an $1 \mathrm{wt} \% \mathrm{G1-PVA} 100$ thin film at various humidities. b) Correlation of the PL intensity at $525 \mathrm{~nm}$ versus humidity. The black line is the linear fitting curve. The excitation wavelength was $365 \mathrm{~nm}$.

real applications, such as a water indicator for fuel or organic solvents (see Figure S4) and residual-water monitoring in root-canal treatment in dentistry (see Figure S5).

In summary, we successfully developed a highly efficient purely organic RTP system with an amorphous polymer matrix by introducing rationally designed strong intermolecular interactions. Through systematic investigation, we dem- a)

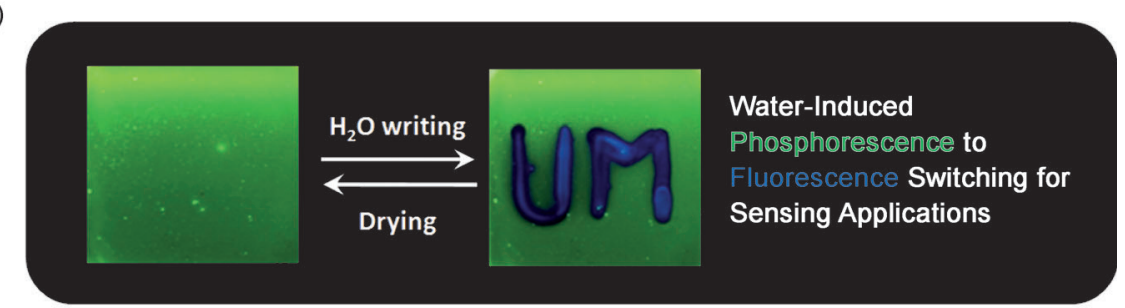

b)

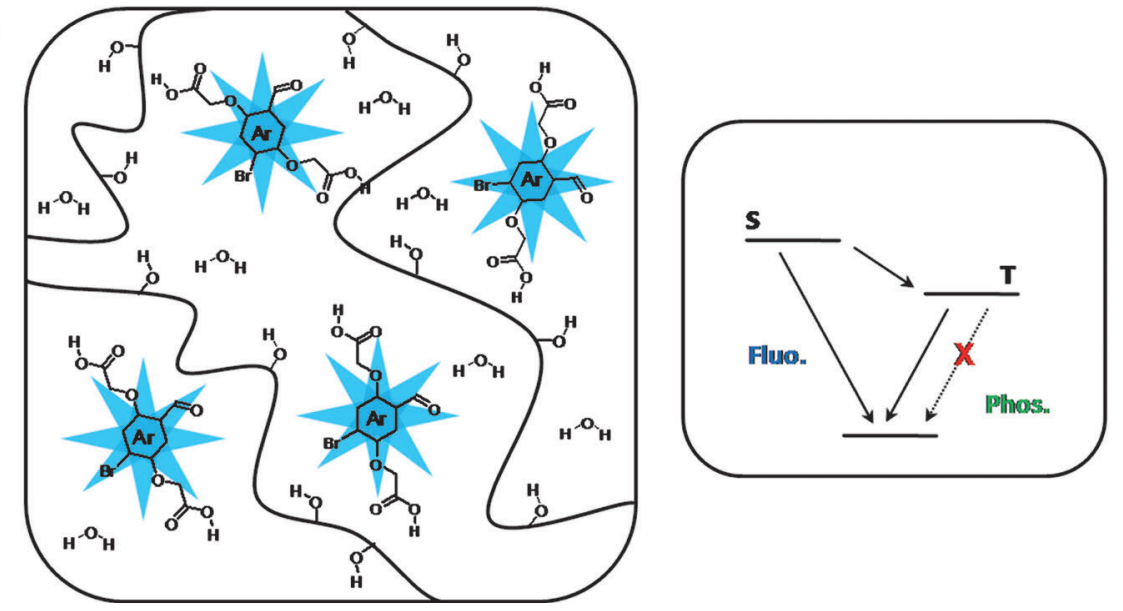

Figure 6. a) Reversible direct writing of a fluorescent watermark on a PVA film embedded with G1. The characters "UM" were written with a water-soaked pen. b) Schematic illustration of phosphorescence processes in the G1-PVA composite. 
onstrated that strong noncovalent interactions, that is, hydrogen and halogen bonds, between the newly designed organic phosphor (G1) and the PVA matrix efficiently suppress the vibrational dissipation of triplet electrons and thus allow a high phosphorescence quantum yield of $24 \%$. We also discovered a unique phosphorescence-to-fluorescence switching phenomenon in the G1-PVA system in response to water. Because, RTP is emitted from a molecular assembly in the G1-PVA system, when water molecules break up the molecular assembly of G1-PVA, the efficiency of spin-orbit coupling drops, thus resulting in fluorescence rather than phosphorescence emission. We believe that the demonstrated strategy based on intermolecular interaction to enable bright RTP from a purely organic material can be utilized for the development of novel phosphors for ratiometric water sensing as well as phosphorescent organic light-emitting diodes for real applications.

Received: April 19, 2014

Published online: July 14, 2014

Keywords: halogen bonds · hydrogen bonds . purely organic phosphors .

room-temperature phosphorescence $\cdot$ water sensors

[1] V. Sivasubramaniam, F. Brodkorb, S. Hanning, H. P. Loebl, V. van Elsbergen, H. Boerner, U. Scherf, M. Kreyenschmidt, $J$. Fluorine Chem. 2009, 130, 640-649.

[2] D. R. Kearns, W. A. Case, J. Am. Chem. Soc. 1966, 88, $5087-$ 5097.

[3] W. D. K. Clark, A. D. Litt, C. Steel, J. Am. Chem. Soc. 1969, 91, $5413-5415$.

[4] J. Xu, A. Takai, Y. Kobayashi, M. Takeuchi, Chem. Commun. 2013, 49, 8447-8449.

[5] E. M. Schulman, C. Walling, Science 1972, 178, 53-54.
[6] L. J. C. Love, M. Skrilec, J. G. Habarta, Anal. Chem. 1980, 52, $754-759$.

[7] C. A. Mitchell, R. W. Gurney, S. H. Jang, B. Kahr, J. Am. Chem. Soc. 1998, 120, 9726-9727.

[8] M. W. Dewhirst, C. L. Fraser, Nat. Mater. 2009, 8, 747-751.

[9] N. J. Turro, Modern Molecular Photochemistry, University Science Books, Sausalito, 1991, pp. 99-100.

[10] A. Köhler, J. S. Wilson, R. H. Friend, Adv. Mater. 2002, 14, $701-$ 707.

[11] W. Z. Yuan, X. Y. Shen, H. Zhao, J. W. Y. Lam, L. Tang, P. Lu, C. Wang, Y. Liu, Z. Wang, Q. Zheng, J. Z. Sun, Y. Ma, B. Z. Tang, J. Phys. Chem. C 2010, 114, 6090-6099.

[12] O. Bolton, K. Lee, H. J. Kim, K. Y. Lin, J. Kim, Nat. Chem. 2011, 3, 205-210.

[13] Y. Gong, Y. Tan, H. Li, Y. Zhang, W. Yuan, Y. Zhang, J. Sun, B. Z. Tang, Sci. China Chem. 2013, 56, 1183-1186.

[14] G. Bergamini, A. Fermi, C. Botta, U. Giovanella, S. Di Motta, F. Negri, R. Peresutti, M. Gingras, P. Ceroni, J. Mater. Chem. C 2013, 1, 2717-2724.

[15] H. Y. Gao, X. R. Zhao, H. Wang, X. Pang, W. J. Jin, Cryst. Growth Des. 2012, 12, 4377-4387.

[16] K. Horie, I. Mita, Chem. Phys. Lett. 1982, 93, 61-65.

[17] K. Horie, K. Morishita, I. Mita, Macromolecules 1984, 17, $1746-$ 1750.

[18] S. Hirata, K. Totani, J. Zhang, T. Yamashita, H. Kaji, S. R. Marder, T. Watanabe, C. Adachi, Adv. Funct. Mater. 2013, 23, 3386-3397.

[19] D. Lee, O. Bolton, B. C. Kim, J. H. Youk, S. Takayama, J. Kim, J. Am. Chem. Soc. 2013, 135, 6325-6329.

[20] V. V. Khutoryanskiy, G. Staikos, Hydrogen-Bonded Interpolymer Complexes: Formation, Structure and Application, World Scientific, Singapore, 2009.

[21] S. Nakanishi, Y. Miyawaki, M. Nishikawa, M. Amano, S. Fujiwara, M. Jitou, H. Itoh, M. Kawase, J. Chem. Phys. 1994, $100,3442$.

[22] S. Sreeja, S. Sreedhanya, N. Smijesh, R. Philip, C. I. Muneera, J. Mater. Chem. C 2013, 1, 3851-3861.

[23] R. F. Bhajantri, V. Ravindrachary, A. Harisha, V. Crasta, S. P. Nayak, B. Poojary, Polymer 2006, 47, 3591-3598. 